\title{
Cardiac Bypass Surgery in a Patient with an Indolent Systemic Mastocytosis
}

\author{
Stefan Beckers ${ }^{1 *}$, Vincent Umbrain ${ }^{1}$, Carla Van Gompel ${ }^{1}$, Adriaan Sablon ${ }^{1}$, Elisabeth De Waele ${ }^{2}$, Christian \\ Verborgh $^{1}$, Jan Nijs ${ }^{3}$ and Jan Poelaert ${ }^{1}$ \\ ${ }^{1}$ Department of Anesthesia and Perioperative Medicine, University Hospital Brussels, Belgium \\ ${ }^{2}$ Department of Intensive Care, University Hospital Brussels, Belgium
}

${ }^{3}$ Department of Cardiac Surgery, University Hospital Brussels, Belgium

Submission: December 15, 2017; Published: January 03, 2018

*Corresponding author: Stefan Beckers, Department of Anesthesia and Perioperative Medicine, University Hospital Brussels, Belgium, Email: Stefan.Beckers@uzbrussel.be

Keywords: Mastocytosis; Cardiac Bypass Surgery ; Cardiac Anesthesia ; Cardiovascular Collapse

\section{Introduction}

Mastocytosis is a heterogeneous group of hematologic disorders with serious anesthetic implications. It is characterized by mast cell accumulation and proliferation in one or more organs, preferentially in the skin and in the bone marrow. Release of histamine and other mast cell mediators (tryptase, chymase, TNF $\alpha$, IL 1, IL 6) can lead to cardiovascular collapse and even to death. We report successful perioperative management of a patient with a known indolent systemic mastocytosis, without any surgical antecedents scheduled for CABG (Cardiac Artery Bypass Grafting).

\section{Case Presentation}

A 57-year-old male with a medical history of 10 years of indolent systemic mastocytosis, presented for elective CABG. He had a history of arterial hypertension, obesity and smoking. Since 2 years, urticaria pigmentosa on his back augmented with many scratch lesions. A bone marrow biopsy was done, showing a D816V-mutation of the C-KIT proto-oncogen. There was no clear history or symptom of allergy. The serum tryptase level was fluctuating, but rising progressively over time. The level from last year was 76 microgram/l. In a general adult population, a median serum tryptase level is 5,1 microgram/l. Actual symptoms were thoracic pain, dyspnoea and diffuse pruritus over his body. His current medications included tritrace, aerius, cetirizine and lercanidipine hydrochloride. He denied a history to drug allergies or allergy to local anesthetic agents. Besides his arterial hypertension and obesity, he had also a familial predisposition for cardiovascular disease. Due to his mastocytosis, he was sent multidisciplinary to an allergist and to an anesthetist for a full examination. Before admission to the hospital, there was a multidisciplinary consultation between an anesthetist, an allergist, a cardiac surgeon and an intensive care physician.
The patient was admitted to the hospital 24 hours preoperatively. He received prednisone $50 \mathrm{mg}$ and levocetirizine $10 \mathrm{mg}$ PO. Twelve hours preoperatively, he received prednisone $50 \mathrm{mg}$ PO, levocetirizine $10 \mathrm{mg}$ PO, ranitidine $150 \mathrm{mg}$ PO and montelukast $10 \mathrm{mg}$ PO. Eight hours before surgical intervention, he was fasted. Two hours before intervention he received prednisone $50 \mathrm{mg}$ PO, levocetirizine $10 \mathrm{mg}$ PO, ranitidine $150 \mathrm{mg}$ PO, montelukast $10 \mathrm{mg}$ PO and lercadipine hydrochloride $10 \mathrm{mg}$ PO. One hour preoperatively, the patient received promethazine hydrochloride $50 \mathrm{mg}$ IM. Then surgery was performed in a latexfree environment per routine hospital practice. After installation and monitoring of ECG, pulse oximetry and noninvasive blood pressure, the patient received a peripheral venous catheter and a radial arterial catheter under local anesthesia. General anesthesia was induced with midazolam ( $3 \mathrm{mg}$ ), propofol (250mg), sufentanil (20microgram) and cisatracurium (14mg) intravenously. Antibiotic prophylactic coverage was done with cefazoline $2 \mathrm{~g}$ intravenously after endotracheal intubation. Maintenance was done with sevoflurane (2\%ET) and with a continuous infusion of sufentanil keeping a NeuroSense (NeuroWave) from 40 to 60 . Cerebral oximetry was also installed on the patient's head to obtain NIRS. A deep central line was inserted in the right internal jugular vein without any problems. During the final installation for surgery and during the incision, the patient remained remarkably stable.

The transoesophageal echocardiography examination after induction was quite normal showing no regional wall motion abnormabilities and borderline normal global left heart contractility. The patient remained hemodynamically stable, without erythema. Then he received heparine $4 \mathrm{mg} /$ $\mathrm{kg}$ ) intravenously before the start of the Extracorporeal Circulation. The surgeon performed two bypasses: left internal 
mammary artery on left anterior descendens artery and a vein on the circumflex artery under normothermia. During the bypass procedure the patient remained stable receiving sevoflurane (between 1 and 2\%ET) for maintenance. The perfusion pressure was kept around $60 \mathrm{mmHg}$ by the perfusionist with a DO2i of 300 $\mathrm{ml} 02 / \mathrm{min} / \mathrm{m}^{2}$. After an ECC of 77 minutes and an aortic clamp of $23 \mathrm{~min}$ with a reperfusion of $50 \mathrm{~min}$, the patient was weaned of bypass. Checklist was performed. Blood gases revealed th that moment a pH of 7.315, a BE of $-2.7 \mathrm{mEq} / \mathrm{L}$, paO2 $155 \mathrm{mmHg}$, paCO2 45,7 mmHg, Hb 11, 3g/dl, lact 1,1 mmol/l, K 5, 6mEq/L and glycemia $161 \mathrm{mg} / \mathrm{dl}$. The patient performed wonderfully well till 5 minutes. Then suddenly his blood pressure crashed down together with ventricular extrasystoles and later on ventricular tachycardia (VT). The patient was shocked internally two times with 30 Joules and became once again regular. Heparin was given for a second run to go on pump again.

Amiodarone (300 mg IV) was given slowly followed by a continuous infusion of $900 \mathrm{mg}$ iv over 24 hours. Echocardiography was done on pump immediately, showing no abnormal regional wall motion. The bypasses were checked by the surgeon and were without any problems. Bilateral breath sounds were auscultated and there was no sign of urticaria or erythematous rash on the visage. The patient became rather vasoplegic and hypotensive and so he received boluses of phenylephrine (100 microgram) followed by a continuous infusion of norepinephrine intravenously $(0,15 \mathrm{microgram} / \mathrm{kg} / \mathrm{min})$. The patient was stabilized and after a second reperfusion of 30 minutes he was weaned of bypass uneventfully. Then he received slowly protamine hydrochloride intravenously for heparine antagonisation. During the postoperative course, he remained under norepinephrine and amiodarone the first postoperative day. On intensive care, propofol was given for sedation and fentanyl for analgesia. He received also cetirizine $10 \mathrm{mg}$, ranitidine $150 \mathrm{mg}$, montelukast 10 $\mathrm{mg}$ and hydrocortisone $40 \mathrm{mg}$ all intravenously. He was weaned of the tube uneventfully in the night after the intervention. Norepinephrine infusion was gradually stopped and the patient was stable and comfortable with fentanyl and paracetamol. He had no cognitive dysfunction and he went to the ward after 3 days. He left the hospital after 10 days. He gave his written informed consent for the publication of this case report.

\section{Discussion}

Indolent Systemic Mastocytosis (ISM) [1,2] is the most common form of systemic mastocytosis, comprising about two thirds of all cases. It involves mainly the skin and the bone marrow and it is diagnosed in adults. Most patients with CM (Cutaneous mastocytosis) and ISM remain in an indolent (sleeping) stage over many years or even decades, while others in particular those with aggressive forms of SM show a progressive course, usually with a fatal outcome. We report a case of Indolent Systemic Mastocytosis, operated for elective CABG. To our knowledge only three cases of patients with systemic mastocytosis undergoing cardiac surgery are reported. Mastocytosis is a rare clinical condition with an estimated incidence of $1 / 150000$ [3]. the cumulative incidence of anaphylaxis in adult patients with mastocytosis is as high as $49 \%$. Actually, there is a dearth of literature on preoperative management in the setting of systemic mastocytosis, mostly consisting of isolated case reports in cardiac surgery [4-6]. In fact, there are no clear guidelines for safe surgery for those patients requiring cardiac surgery. In 2009, Chaar [7] proposed guidelines for the perioperative management of these patients, based on their own experiences. Selective skin testing, continuation of daily medications, administration of antihistamines and corticosteroids and maintaining anesthesia with inhalational agents are the main recommendations. Prior treatment with ant-H1, ant-H2, corticosteroids and sodiumcromoglycaat is to put the mast cells at rest, to prevent degranulation. But despite pretreatment, significant hypotension [4] and even cardiac arrest can develop $[8,9]$.

The recommendations followed are based upon experience of other groups, previous case reports and recommendations from allergy, dermatology and hematology colleagues. Surgery per se may trigger extensive mast cell degranulation. It can be due to the administration of drugs or to the procedure itself. So, a good plan for anesthesia is careful and optimal planning of the intervention from the time of the preoperative visit, to the surgery as well as the postoperative period. Good communication is essential and anxiolysis is recommended because anxiety may precipitate mast cell degranulation. A quiet operating room is preferred when dealing with such patients [3]. The Ring and Messmer's four-step grading scale is appropriate to describe the clinical severity of immediate perioperative hypersensitivity [10]. Grade I reactions involve only mucocutaneous signs and symptoms, grade II reactions correspond to mild mucocutaneous features associated with cardiovascular and/or respiratory signs. Cardiovascular collapse [9] is the cardinal sign of grade III reaction, associated with bronchospasm, mucocutaneous or gastrointestinal signs. Grade IV reactions present with cardiac arrest. Grade I and II are non life-threatening conditions, grade III and IV necessitate urgent resuscitation.

In general cardiovascular signs do not correlate with the cutaneous or systemic phenotype of the disease. In this patient, we didn't perform prior skin testing, because he had no prior exposure to anesthetic agents, so he had not the time to form antibodies. The last known tryptase level preoperatively was 76 microgram/l and this dated from the year before. He had a stable form of indolent systemic mastocytosis with emphasis on cutaneous symptoms, followed annually by an expert hematologist. The skin infiltration expanded over the years what resulted in a fluctuation and a slow augmentation of the tryptase level. Because there is no routine measurement of the tryptase level in our hospital, we didn't perform it during and after the procedure. We pay attention to a good preoperative and multidisciplinary consultation. We asked preoperatively the advice of an allergist, of an intensivist, of a cardiac surgeon and a cardiac anesthetist to prepare maximally the patient for this intervention. The main purpose was to put on one hand the mast cells at rest by giving the patient his routine medication and on the other hand to 
control and to stabilize the heart during the perioperative traject. Each member of the cardiac team was aware of the fact that the patient could crash on one moment or another. The patient was admitted 24 hours preoperatively to be well prepared for the operation. Meanwhile he was put maximally under histamines, montelukast and prednisone. Finally, he didn't show allergic reactions till the weaning of the extracorporeal circulation after 30 minutes of reperfusion. The first 5 minutes after the first run were uneventful and then suddenly the patient collapsed and did ventricular extrasystoles and VT. He became also vasoplegic. He needed a second run and a second reperfusion with amiodarone and norepinephrine, and only once stabilized, the patient could be weaned easily. Inspiratory pressures were not elevated.

The extrasystoles disappeared but the vasoplegia status which happened after the VT period, remained and necessitated a norepinephrine infusion up to the intensive care room. The patient tolerated well the protaminehydrochloride injection. In the second run the surgeons exclude a problem of the bypass patency and also on TOE there was no air seen in the left ventricle (nor before, nor after the first run, excluding air in the bypasses) and thus provoking arrhythmia and ventricular tachycardia. Contractility was normal and homogeneous in all segments as judged by the anesthesiologist. Cardiac surgery can lead to a greater risk for mast cell degranulation in systemic mastocytosis than general surgical procedures (with the exception of abdominal surgery). The artificial surface of the CPB, the aortic cross clamping, the aortic declamping, the ischemia-reperfusion injury, the operative trauma, as well as temperature dysregulation (not in this case, because the patient was operated under normothermia) can lead to mast cell degranulation [4]. The weaning of the bypass was probably a trigger for mast cell degranulation and in fact this was not documented by a tryptase level measurement. The weaning is a particular part of ECC with possible pharmacological, mechanical and temperature changes, even when it is done gently and smoothly by a careful perfusionist. The patient responded well to norepinephrine infusion and to amiodarone infusion and after a second reperfusion, he was weaned successfully without contractility problems. Inspiratory pressures (indicating bronchospasm) were not elevated and there was no erythematous rash on the skin. In general, bronchospasm usually does not occur
$[11,12]$. In summary we describe a successful management of a patient undergoing a CABG with antecedents of an indolent systemic mastocytosis. This patient presented a serious reaction with cardiovascular collapse on the moment of the first weaning of bypass necessitating a second run and a norepinephrine and amiodarone infusion. Intraoperative mast cell degranulation may result in a hypersensitivity reaction in patients with diagnosed mastocytosis despite prior pretreatment.

\section{References}

1. Horny HP, Metcalfe DD, Bennett JM (2008) WHO Classification of Tumours of Haemotopoetic and Lymphoid Tissues. IARC Press, Lyon, France, p. 54-63.

2. Matito A, Morgado JM (2015) Management of anesthesia in adult and pediatric mastocytosis: a study of the Spanish Network on Mastocytosis (Rema) based on 726 anesthetic procedures. Int Arch Allergy Immunol 167(1): 47-56.

3. Pascale Dewachter, Mariana Casttells (2014) Perioperative Management of patients with mastocytosis. Anesthesiology 120: 753759.

4. Neal Duggal, Erin Payne Milo Engoren (2015) Aneurysm Repair in a Patient With Systemic Mastocytosis. Journal of Cardiothoracic and Vascular Anesthesia 29(4): 1025-1028.

5. Moro JA (2008) Heart transplantation in a patient with systemic mastocytosis. J Heart Lung Transplant 27(6): 689-691.

6. Damodar S (2006) Mast cell disease Surgical and anesthetic implications. J Pediatric Hematol Oncol 28(7): 446-449.

7. Chaar CI, Bell RL, Duffy TP (2009) Guideliness for safe surgery in patients with systemic mastocytosis. Am Surg 75(1): 74-80.

8. Renaud V (2011) Case Report. Perioperative immediate hyper sensitivity involves not only allergy but also Mastocytosis. Can J Anaesthesia 58: 456-459.

9. Vaughan ST, GN Jones (1998) Systemic Mastocytosis presenting as profound cardiovascular collapse during anesthesia. Anesthesia 53(8): 804-807.

10. Horny HP, Sotlar K, Valent P (2007) Mastocytosis: State of the Art. Pathobiology 74(2):121-132.

11. Borgeat, Ruetsch, Yvan A (1198) Anesthesia in a Patient with Malignant Systemic Mastocytosis using a Total Intravenous anesthetic Technique. Anesthesia \& Analgesia 86(2): 442-444.

12. Desborough JP, Taylor I, Hattersley A, Garden A, Wolff A, et al. (1990) Massive histamine release in a patient with systemic mastocytosis. $\mathrm{Br}$ J Anaesth 65(6): 833-836.

\begin{tabular}{|l|}
\hline \multicolumn{1}{|c|}{ Your next submission with Juniper Publishers } \\
will reach you the below assets \\
- Quality Editorial service \\
- Swift Peer Review \\
- Reprints availability \\
- E-prints Service \\
- Manuscript Podcast for convenient understanding \\
- Global attainment for your research \\
- Manuscript accessibility in different formats \\
( Pdf, E-pub, Full Text, Audio) \\
- Unceasing customer service \\
Track the below URL for one-step submission \\
https://juniperpublishers.com/online-submission.php \\
\hline
\end{tabular}

